\title{
Manifestation of echocardiography for dynamic change in right ventricular function and structure of patients with pulmonary hypertension.
}

\author{
Zhao Ping* \\ Ultrasonic Lab, Shangluo Central Hospital, No. 148 Beixin Street, Shangluo, Shaanxi, PR China
}

\begin{abstract}
Objective: To analyze influence of the function and the structure of right pulmonary artery hypertension patients by echocardiography.

Methods: A total of 50 patients with pulmonary hypertension accepted by our hospital from March 2013 to April 2014 were selected by random number table, and randomly selected 50 healthy people as control group for medical examination in our hospital in the same period. Echocardiography examination was implemented for all patients. Recorded and compared two groups of patients with their tricuspid regurgitation area (ATR), the inner diameter of the inferior vena cava (DIVC), right ventricular end-diastolic volume (EDV), right ventricular end-systolic volume (ESV), right ventricular ejection fraction (RV-EF), left ventricular diameter (LVTD), right ventricular diameter (RVTD), pulmonary trunk diameter (DMPA), and right pulmonary artery (DRPA), left pulmonary artery (DLPA), tricuspid annulus systolic displacement (TAPSE), right cardiac work index (RIMP), right ventricular fractional area change (FAC), tricuspid annulus peak systolic velocity ( $\mathrm{s}^{\prime}$ ), tricuspid annulus peak early diastolic velocity ( $\left.e^{\prime}\right)$, tricuspid annulus diastolic Late peak velocity (a'), and calculates RVTD/LVTD, $\mathrm{e}^{\prime} / \mathrm{a}^{\prime}$, left ventricular ejection fraction (LVEF).

Results: The echocardiography showed that right ventricular structure (ATR, RVEDA, RVESA, LVTD, RVTD, DMPA, DRPA, DLPA) of pulmonary hypertension compared with the control group, their difference was statistically significant $(\mathbf{P}<0.05)$; pulmonary right ventricular function (TAPSE, RIMP, FAC, s', e ', a')in pulmonary hypertension patients compared with those on control group, there was significant difference $(\mathrm{P}<0.05)$; and RVTD/LVTD, $\mathrm{e}^{\prime} / \mathrm{a}^{\prime}$, LVEF of pulmonary hypertension were also different from control group $(\mathbf{P}<\mathbf{0 . 0 5})$.

Conclusion: Echocardiography showed that, patients with pulmonary hypertension will lead to decline in right heart dysfunction and enlarge the right heart becomes, echocardiography has a good effect for diagnosis, treatment and prognosis of patients with pulmonary hypertension.
\end{abstract}

\section{Introduction}

Pulmonary artery hypertension (PAH) is the major reason for right heart failure which is also the most significant lethal factor for patients with pulmonary artery hypertension $[1,2]$. The infants born too early have Pulmonary Hypertension (PH) with Broncho-Pulmonary Dysplasia (BPD) such that the range is nearly $14-37 \%$. As high as $38 \%$, it is related with a major death risk. In past years, $\mathrm{PH}$ specific representatives have been used in BPD related PH management and without randomized controlled trials, the survival has been enhanced. The medical and respiratory controlling is adjusted when the $\mathrm{PH}$ is detected at early stage. The screening echocardiograms for $\mathrm{PH}$ are implemented continuously for the infant having high risk.

The patients with PH are examined by a biochemical marker named as N-terminal pro-B-type Natriuretic Peptide (NT-pro$\mathrm{BNP})$. Among premature infants with $\mathrm{PH}$ secondary to BPD, it is not evaluated prospectively. Amino acid signs of Nitric
Oxide (NO) have been associated in the pathogenesis of certain $\mathrm{PH}$ method and embarrassment of endogenous NO production has been recommended to play a role in the BPD related $\mathrm{PH}$ population [3].

Locating Left Heart Disease (LHD) or Heart Failure (HF) is common however its occurrence is underappreciated. Delusions in pathophysiology, diagnosis and management happen when there is a varying terms. The $\mathrm{PH}$ is diagnosed exactly based on hemodynamic assessment through right heart catheterization and complete echocardiography and integrative scoring methods are used regularly because of limitation in contact. The setting of PH owed to LHD, Right Ventricular Dysfunction (RVD) deliberates a poor clinical diagnosis. RVD organization is focused on treating essential HF and/or valvular disease. Longitudinal information is attained by observing implantable hemodynamic for enhancing diagnostic accuracy and to observe effects of $\mathrm{PH}$ treatment in setting of HF with and without the existence of RVD [4]. 
Therefore, timely and correct diagnosis and evaluation of right ventricle change caused by pulmonary artery hypertension is significant to the evaluation, treatment and prognosis of illness condition [5]. In recent years, new technologies, including twodimensional echocardiogram deformation and deformation rate, three-dimensional imaging and Tei index replace and compensate the deficiencies of traditional ultrasound in evaluating right heart structure and function. Our hospital studied and analyzed the echocardiogram manifestation in change of right heart function and structure of pulmonary artery hypertensionpatients.

\section{Data and Methods}

\section{Clinical data}

A total of 50 patients with pulmonary artery hypertension, received by Shangluo Central Hospital from March 2013 to
April 2014 were randomly selected as observation group by random number table, and 50 patients receiving physical examination in our hospital with normal pulmonary artery pressure were randomly selected as control group. Inclusion criteria of patients with pulmonary artery hypertension: 1) the pulmonary arterial systolic pressure of patients $>35 \mathrm{mmHg}$; 2 ) good general condition without severe disease. The whole work was implemented with patients were well informed and approval by ethic committee of our hospital. The age of patients in observation group was $33 \sim 67$, while that in control group was 32 65. Patients in both groups had no statistical significance in sex, age, body mass index and other general data without significant difference $(\mathrm{P}>0.05)$. However, the pulmonary artery pressure and heart rate of patients in observation group were all higher than those in control group $(\mathrm{P}<0.05)$ (Table 1).

Table 1. Comparison of general data between two groups.

\begin{tabular}{|c|c|c|c|c|}
\hline Group & Observation group $(n=50)$ & Control group $(n=50)$ & Statistical value & $P$ value \\
\hline Male/female (n) & $21 / 29$ & $24 / 26$ & 0.3636 & 0.5465 \\
\hline Average age $(\bar{x} \pm s$, years $)$ & $56.2 \pm 7.8$ & $55.8 \pm 8.2$ & 0.2499 & 0.8032 \\
\hline Body mass index $\mathrm{BMI}\left(\overline{\mathrm{x}} \pm \mathrm{s}, \mathrm{Kg} / \mathrm{m}^{2}\right)$ & $24.78 \pm 3.24$ & $24.51 \pm 3.67$ & 0.3900 & 0.6974 \\
\hline hemoglobin $(\bar{x} \pm s, g / L)$ & $142.3 \pm 25.3$ & $138.9 \pm 22.3$ & 0.7129 & 0.4776 \\
\hline Serum creatinine $(\bar{x} \pm s, \mu \mathrm{mol} / L)$ & $85.9 \pm 23.3$ & $77.5 \pm 24.3$ & 1.6572 & 0.1007 \\
\hline Heart rate $/\left(\right.$ times $\cdot \mathrm{min}^{-1}$ ) & $73.3 \pm 8.9$ & $86.8 \pm 12.4$ & 6.2542 & $<0.0001$ \\
\hline Pulmonary artery pressure $(\mathrm{mmHg})$ & $58.0 \pm 12.9$ & $24.4 \pm 6.6$ & 16.3963 & $<0.0001$ \\
\hline Combined hypertension [n (\%)] & $41(82.00)$ & $27(54.00)$ & 9.0074 & 0.0027 \\
\hline
\end{tabular}

\section{Methods}

All the patients rested on bed after being admitted to hospital to stabilize vital signs and maintain breath and cycle. Threedimensional cardiac ultrasound diasonograph (manufacturer: Philips iE33) equipped with the probe for real-time threedimensional cardiac ultrasound (type: X3-1 matrix) was used, and the probe frequency is $22.5 \sim 3.5 \mathrm{MHz}$. Workstation for processing ultrasonoscopy of TomTec Research-Arena was used, and 4DRV-function software in it was used for analysis. When implementing the examination by three-dimensional cardiac diasonograph, subjects took left lateral position to record electrocardiogram. After adjustment, patients were asked to hold breath. Probe was placed on heart of subject to obtain ideal image in apical four-chamber view. Then, probe direction was adjusted slightly to make right ventricle at the center of the image. Full-volume imaging modality was restarted to collect "pyramid-type" three-dimensional database of continuous cardiac cycle by automatic trigger of cardiogram. Collected three-dimensional image was stored in hard disc, and then uploaded to workstation TomTec after examination for analysis with 4DRV-function software. Tricuspid regurgitation (ATR), inner diameter of postcava (DIVC), right ventricular end diastolic volume (EDV), right ventricular end systolic volume (ESV), right ventricular ejection fraction (RV-EF), left ventricular transverse diameter (LVTD), right ventricular transverse diameter (RVTD), inner diameter of main pulmonary artery (DMPA), inner diameter of right pulmonary artery (DRPA), inner diameter of left pulmonary artery (DLPA), systolic mitral annular displacement (TAPSE), right cardiac work index (RIMP), right ventricular fractional area change (FAC), peak systolic velocity of tricuspid valve (s'), early diastolic myocardial peak velocity of tricuspid valve (e') and peak velocity in late diastole of tricuspid valve (a') of patients in both groups were recorded and compared. Then, RVTD/LVTD, e'/a' and left ventricular ejection fraction (LVEF) were calculated.

For Statistics, SPSS18.0 was chosen and data was denoted with mean \pm standard deviation $(\overline{\mathrm{x}} \pm \mathrm{s})$. Measurement data was compared with $\mathrm{t}$ test, ranked data with rank sum test, and enumeration data with $\chi^{2}$ test. $\mathrm{P}<0.05$ indicated that the data differences were statistically significant. 


\section{Results}

\section{Comparison of parameters for right ventricular structure between both groups}

Ultrasonic cardiogram shows that the right ventricular structure of patients with pulmonary artery hypertension (ATR, DIVC,
EDV, ESV, LVTD, RVTD, DMPA, DRPA, DLPA and LVTD/ RVTD) had difference with statistical significance compared with normal control group $(\mathrm{P}<0.05)$ (Table 2).

Table 2. Parameter comparison for right ventricular structure among two groups $(\bar{x} \pm s)$.

\begin{tabular}{|c|c|c|c|c|c|}
\hline Group & $\operatorname{ATR}\left(\mathrm{cm}^{2}\right)$ & DIVC (mm) & $\mathrm{EDV} / \mathrm{ml}$ & $\mathrm{ESV} / \mathrm{ml}$ & LVTD (mm) \\
\hline Observation group $(n=50)$ & $2.6 \pm 0.4$ & $16.3 \pm 3.2$ & $194.1 \pm 32.2$ & $136.3 \pm 33.7$ & $31.23 \pm 3.12$ \\
\hline Control group $(n=50)$ & $0.6 \pm 0.2$ & $11.1 \pm 2.1$ & $81.9 \pm 15.9$ & $31.1 \pm 11.2$ & $35.78 \pm 1.88$ \\
\hline $\mathrm{t}$ value & 26.7214 & 9.6066 & 22.0924 & 20.947 & 8.8324 \\
\hline$P$ value & $<0.0001$ & $<0.0001$ & $<0.0001$ & $<0.0001$ & $<0.0001$ \\
\hline Group & RVTD (mm) & DMPA (mm) & DRPA $(\mathrm{mm})$ & DLPA (mm) & LVTD/RVTD \\
\hline Observation group $(n=50)$ & $45.89 \pm 8.88$ & $31.89 \pm 6.56$ & $23.34 \pm 3.43$ & $20.54 \pm 3.33$ & $1.55 \pm 0.45$ \\
\hline Control group $(n=50)$ & $31.03 \pm 2.14$ & $23.67 \pm 2.34$ & $16.89 \pm 1.99$ & $17.15 \pm 2.39$ & $0.88 \pm 0.07$ \\
\hline $\mathrm{t}$ value & 11.5036 & 8.3454 & 11.5014 & 5.8481 & 10.4029 \\
\hline$P$ value & $<0.0001$ & $<0.0001$ & $<0.0001$ & $<0.0001$ & $<0.0001$ \\
\hline
\end{tabular}

\section{Comparison of parameters for right ventricular function between two groups}

Compared with normal control group, the right ventricular functions of patients with pulmonary artery hypertension (TAPSE, RIMP, FAC, s', e', a') had difference with statistical significance $(\mathrm{P}<0.05)$. Moreover, RV-EF, e'/a' and LVEF of patients with pulmonary artery hypertension also became abnormal with difference with statistical significance $(\mathrm{P}<0.05)$ (Table 3). Ultrasonic cardiogram of patients with pulmonary artery hypertension shows as in Figure 1.

Table 3. Parameter Comparison for Right Ventricular Functions among Two Groups $(x \pm s)$.

\begin{tabular}{|c|c|c|c|c|c|c|c|c|c|}
\hline Group & TAPSE $(\mathrm{mm})$ & RIMP & FAC (\%) & $\mathrm{s}^{\prime}(\mathrm{cm} / \mathrm{s})$ & $e^{\prime}(\mathrm{cm} / \mathrm{s})$ & $a^{\prime}(\mathrm{cm} / \mathrm{s})$ & $e^{\prime} / a^{\prime}$ & LVEF (\%) & RV-EF/\% \\
\hline $\begin{array}{l}\text { Observation } \\
\text { group }(n=50)\end{array}$ & $14.43 \pm 2.07$ & $0.88 \pm 0.31$ & $29.33 \pm 4.78$ & $11.89 \pm 3.38$ & $9.69 \pm 2.54$ & $16.10 \pm 3.48$ & $0.75 \pm 0.34$ & $72.28 \pm 6.16$ & $30.3 \pm 5.4$ \\
\hline $\begin{array}{l}\text { Control group } \\
(n=50)\end{array}$ & $17.01 \pm 1.16$ & $0.41 \pm 0.12$ & $43.42 \pm 3.21$ & $17.91 \pm 3.39$ & $12.10 \pm 2.68$ & $10.78 \pm 2.89$ & $1.13 \pm 0.26$ & $66.54 \pm 3.89$ & $60.6 \pm 7.8$ \\
\hline $\mathrm{t}$ value & 7.6883 & 9.9977 & 17.3037 & 8.8922 & 4.6152 & 8.3161 & 6.2778 & 5.5711 & 22.5843 \\
\hline$P$ value & $<0.0001$ & $<0.0001$ & $<0.0001$ & $<0.0001$ & $<0.0001$ & $<0.0001$ & $<0.0001$ & $<0.0001$ & $<0.0001$ \\
\hline
\end{tabular}

\section{Discussion}

In recent years, medical workers pay more and more attention to problems of diagnosis, treatment and evaluation of pulmonary artery hypertension [6-8]. Once upon patients with pulmonary artery hypertension has clinical symptoms, the most symptoms show the right ventricular functions and severe injury. Some researches show that 3-year survival rate of patients with pulmonary artery hypertension for different pathogen is only $20 \% \sim 70 \%$ [8-10]. Prognosis of pulmonary artery hypertension for different pathogens is different. Some researches show that the prognosis of pulmonary artery hypertension caused by congenital heart disease is always good, and its 3 year survival rate can be up to $30 \%$. The prognosis of pulmonary artery hypertension caused by human immunodeficiency virus and connective tissue diseases is always bad, and 3 year survival rate is only $20 \% \sim 40 \%$. Prognosis of pulmonary artery hypertension caused by unknown reason is worse, and the average survival year from disease diagram is only 2.8 years [4.11]. With the deepening of cognition for the importance of right ventricular functions, it is particularly important to seek a simple, feasible, non-invasive, correct and well repeatable way to evaluate right heart. Realtime three-dimensional cardiac ultrasound effectively overcomes the limit of application range of two-dimensional ultrasound in clinical, and can directly make quantitative measurement of actual ventricular volume. This measurement has higher accurate and convenient measurement of irregular geometrical ventricular volume which can satisfy with accurate evaluation of right ventricular functions in clinical [11-13]. 

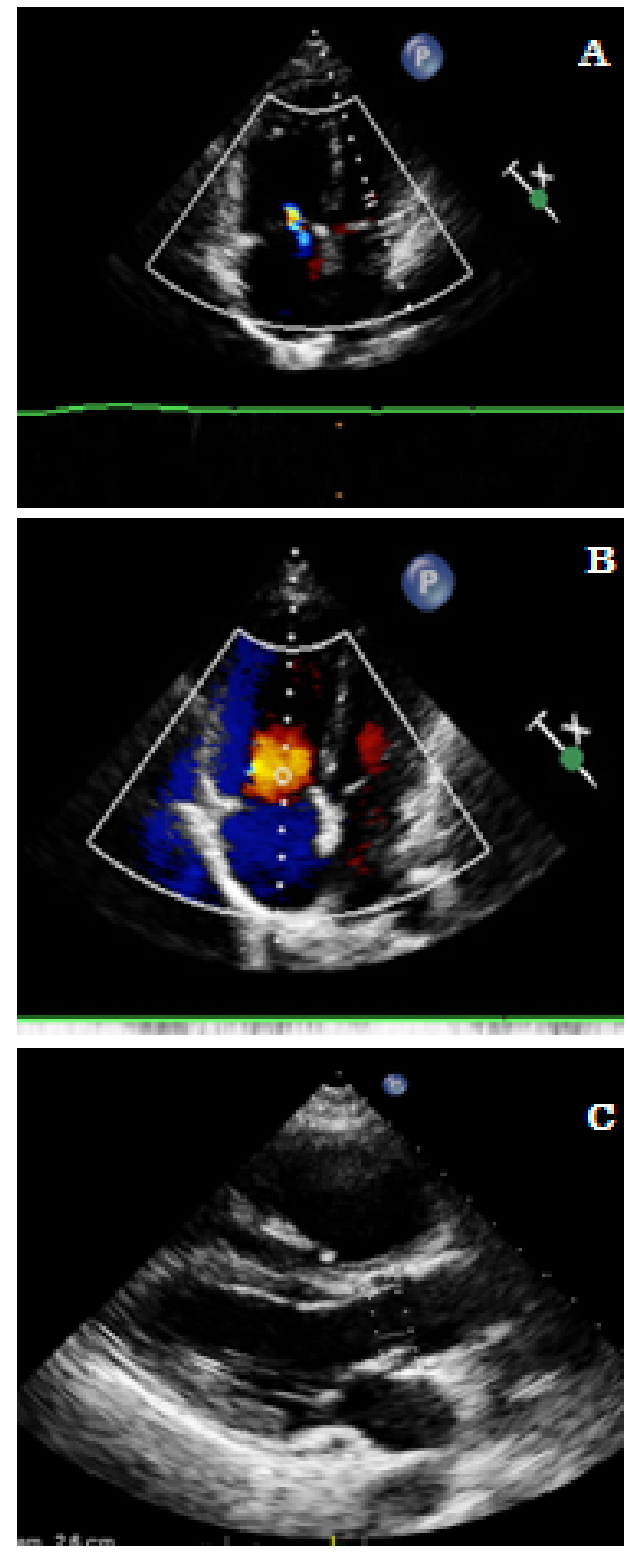

Figure 1. A and B show significant tricuspid regurgitation; $C$ shows significant thickening of right ventricle.

PAH may cause the afterload increase in right ventricle to make it have compensatory reconstruction. Therefore, the length of myocardial cell may increase, the wall of right ventricle may thicken, collating sequence of sarcomere may disorder, and interstitial fibrosis and fibroplasia may occur under endocardium and around vessel, respectively. Lamberts et al. induced hypertrophy of rat right ventricle with monocrotaline to establish a model. PAH formed accompanying with right ventricular hypertrophy 28 days after injection, and the change of right ventricular change may eventually cause the diastolic hypofunction of left ventricle. Therefore, when PAH occurred, the structure of right ventricle may change, and the failure of cardiac function may occur. Some researches show that main pulmonary artery (MPA) may broaden, pulmonary arterial systolic pressure (PASP) may significantly increase, left-right diameter at diastole end of right ventricle (RVED) may enlarge, and regurgitation velocity of tricuspid valve may be faster among patients with PAH [14]. Our hospital studied and analyzed the ultrasonic cardiogram of dynamic change in right ventricular function and structure of patients with pulmonary artery hypertension. Some researchers showed that in ultrasonic cardiogram, compared with normal control group, the right ventricular structure of patients with pulmonary artery hypertension (ATR, DIVC, EDV, ESV, LVTD, RVTD, DMPA, DRPA and DLPA) has significant change. ATR, DIVC, EDV, ESV, RVTD, DMPA, DRPA, DLPA and LVTD/RVTD all have significant increase, while LVTD has significant decrease. It means that ultrasonic cardiogram has good diagnosis effects on the change of right ventricular structure.

Sukmawan et al. found that patients with PAH had significant increase in volume at systolic end and diastasis when observing the right ventricular form of patients with threedimensional ultrasonic cardiogram. It implies that the change in geometrical structure of right ventricle may further develop into right cardiac insufficiency to make right ventricular function increase when right ventricle has significant thickening and expansion [15]. Our hospital found that compared with normal control group, the right ventricular function of patients with pulmonary artery (TAPSE, RIMP, FAC, s', e' and a') had significant abnormality, and RV-EF and e'/a' decreased. It means that pulmonary artery hypertension causes the decrease of right ventricular function of patients.

In conclusion, ultrasonic cardiogram shows the decrease in patients' right ventricular function with pulmonary hypertension and enlargement of right heart. Ultrasonic cardiogram may have key role in the diagnosis and judgment prognosis of patients with pulmonary artery hypertension.

\section{References}

1. Fetzer DT, Rees MA, Dasyam AK, Tublin ME. Hepatic sarcoidosis in patients presenting with liver dysfunction: imaging appearance, pathological correlation and disease evolution. European Radiol 2016.

2. Montgomery AM, Bazzy-Asaad A, Asnes JD, Bizzarro MJ, Ehrenkranz RA, Weismann CG. Biochemical Screening for Pulmonary Hypertension in Preterm Infants with Bronchopulmonary Dysplasia. Neonatology 2016; 109: 190-194.

3. Ota C, Kimura M, Kure S. ABCA3 mutations led to pulmonary fibrosis and emphysema with pulmonary hypertension in an 8yearold girl. Pediatric Pulmonology 2016.

4. Lala A, Pinney SP. Recognizing Pulmonary Hypertension and Right Ventricular Dysfunction in Heart Failure. Progress Cardiovascular Diseases 2016; 58: 416-424.

5. Ghio S, Schirinzi S, Pica S. Pulmonary arterial compliance: How and why should we measure it?. Global Cardiol Sci Practice 2015.

6. Said K. AMBITION: An important piece in the therapeutic puzzle of pulmonary arterial hypertension. Global Cardiol Sci Practice 2015. 
7. Corris PA. The UK National Pulmonary Hypertension Service, Registry and Research Collaboration. Global Cardiol Sci Practice 2015.

8. Chakrabarti AM, Mitchell JA, Wort SJ. Progress in the understanding and management of pulmonary arterial hypertension. Global Cardiol Sci Practice 2015.

9. Linga KR, Khoor A, Phelan JA, Mira-Avendano I. Pulmonary Vein Stenosis Mimicking Nonspecific Interstitial Pneumonia. Case Reprt Pulmonol 2015.

10. Lakshminrusimha S, Mathew B, Leach CL. Pharmacologic strategies in neonatal pulmonary hypertension other than nitric oxide. In Seminars in perinatology 2016; 40: 160-173.

11. Nakamura K, Asano Y, Taniguchi T, Minatsuki S, Inaba T, Maki H, Takahashi T. Serum levels of interleukin18binding protein isoform a: Clinical association with inflammation and pulmonary hypertension in systemic sclerosis. J Dermatology 2016.

12. Nicolson G, Daley M, Makara M, Beijerink N. Partial anomalous pulmonary venous connection with suspected pulmonary hypertension in a cat. J Veterinary Cardiol 2015; 17: S354-S359.
13. Abraham S, Weismann CG. Left Ventricular EndSystolic Eccentricity Index for Assessment of Pulmonary Hypertension in Infants. Echocardiography 2016.

14. Lala RI, Darabantiu D, Pilat L, Puschita M. Galectin-3: A Link between Myocardial and Arterial Stiffening in Patients with Acute Decompensated Heart Failure? Arquivos brasileiros de cardiologia 2016.

15. Sukmawan R, Watanabe N, Ogasawara Y, Yamaura Y, Yamamoto K, Wada N, Yoshida K. Geometric changes of tricuspid valve tenting in tricuspid regurgitation secondary to pulmonary hypertension quantified by novel system with transthoracic real-time 3-dimensional echocardiography. J Am Soc Echocardiography 2007; 20: 470-476.

\section{*Correspondence to}

Zhao Ping

Ultrasonic Lab

Shangluo Central Hospital

PR China 